\section{The Role of Cell Autophagy in Toxicity Caused by Dental Nanomaterials}

\author{
Yanzhen Liü, Jianfeng Wang\#, Mengyun Jiang, Xianqin \\ Tong, Chaochao Han, Bilal Zahid Ramadan and Limin Wei*
}

School and Hospital of Stomatology, Wenzhou Medical University, Wenzhou 325027, China

\begin{abstract}
Nowadays, nanomaterials are widely used in the field of dental materials. However, more and more evidence shows us that nanomaterials have potential toxic effects on bio-systems. The manufacture, use and disposal of nanomaterials, potential risks and their toxicity mechanisms have important effects on the sustainable development of nanotechnology. Currently, a great deal of studies found that autophagy is an important mechanism of cytotoxicity induced by nanomaterials, but the detailed mechanism has not been fully discovered. In this review, we will take nanocomposite resins, carbon nano, nano titanium dioxide, nano silver, which are commonly used in the field of oral medicine, for instance to summarize the effects of nano materials on cell autophagy . In general, based on the chemical and physical properties of nanometer material itself, NPs can be potentially hazardous via several possible mechanisms, such as oxidative stress, autophagy and lysosome dysfunction and the activation of certain signaling pathways. Significant glutathione depletion and reactive oxygen species generation, which reduced the cellular antioxidant level and caused cytoskeleton disruption. The levels of autophagy related genes, especially the DRAM1 gene and the autophagosome formation-related proteins, were clearly up regulated together with an increase of autophagosome like vacuoles. However, the mechanism of nanotoxicity and cause of protective autophagy is still not clear. In addition, the relationship between NPs induced autophagy and other inherent effect as well as the application in tumor treatment and a lack of common standards still needs further study. A new generation of nano materials worth looking forward to which has better biocompatibility, reduces oxidative stress effect and focuses on biosafety.
\end{abstract}

Keywords: Autophagy; Cell toxicity; Dentistry; Nanomaterials

*Corresponding author: Wei Limin, School and Hospital of Stomatology, Wenzhou Medical University, Wenzhou 325027, China, Tel: +86 13738340921; Email: Iwei525@wmu.edu.cn

Citation: Liu Y, Wang J, Jiang M, Tong X, Han C, et al. (2017) The Role of Cell Autophagy in Toxicity Caused by Dental Nanomaterials. J Nanotechnol Nanomed Nanobiotechnol 4: 011.

Received: December 14, 2016; Accepted: February 25, 2017; Published: March 13, 2017

\section{Nanomaterials}

\section{The overview of nanomaterials}

Nanomaterials are defined as materials composed of unbound particles or particles in an aggregate or agglomerate state with one or more external dimensions with a size ranging from $1 \mathrm{~nm}$ to $100 \mathrm{~nm}$ [1]. Nanomaterials have a lot of free surfaces and interfaces and there is interaction between the various units. Based on the above characteristics, nanomaterials are featured by surface effect, small size effect, quantum size effect, macroscopic quantum tunneling effect and the dielectric confinement effect. Nanoparticles have lots of unpaired atoms, large specific surface area, less surface defects and can occur in conjunction with the polymer stronger physically or chemically. Added to the polymer material, they can improve the ductility, toughness, strength, barrier properties, heat resistance and dimensional stability of the material. They have been widely used in conventional materials, medical equipment, electronic equipment, paint and other industries. However, nanomaterials will affect the organism and produce a series of toxic effects by inducing oxidative stress and inflammatory response mechanisms in the cell, subcellular and protein levels.

\section{The nanomaterials commonly used in dentistry}

Nanometer materials are widely applied in the field of dentistry, including dental restoration, antibacterial, caries treatment, orthodontic, root canal, bonding systems and so on.

Adding nanoparticles to dental restoration materials to improve the performance has been widely used in the clinical field. In view of its unpaired atoms, large specific surface area, less surface defects and occurring in conjunction with the polymer either stronger physically or chemically, the novel resin composite exhibited a strong antibacterial property upon the addition of up to $5 \%$ nano antibacterial inorganic fillers, there by leading to effective caries inhibition in dental application [2]. The mesoporous silica biomaterials have great potential for serving as both a catalyst and carrier in the repair or regeneration of dental hard tissue [3]. Diatomite-based nanocomposite ceramics are good potential candidates for ceramic-based dental materials [4]. Nano silicon dioxide can also give polymers the characteristics of better viscosity, thermal stability, high strength, less volume shrinkage and of course, durability [5].

In clinical endodontics especially root canal and restorative treatments we can see the near future potential of nanoparticles from the application of nanoparticles in the form of solutions for irrigation, medication and as an additive within sealers/restorative materials [6]. GICs can be applied to base plates of Orthodontic and modified denture base well, as which contain chlorhexidine hexametaphosphate nanoparticles and characterize the nanoparticle size shows a stronger antimicrobial activity [7].

As for caries treatment, Hannig $\mathrm{M}$ said that Analysis of in vitro data indicates that apatite nanoparticles might be effective in reversing lesion progression in the outer but not in the deeper part of early caries lesions [8]. 
Nano HA which was used as a direct pulp capping agent, showed no inflammatory changes in majority of the samples in both the study periods, based on the ability of nano HA to produce complete dentinal bridges, favorable cellular and vascular response [6].

When it comes to dentin bonding systems, nano-technology really makes a difference. Porenczuk A compared the shear bond strength and microscopic assessment of failure modes for a glass-ionomer cement and dentin bonding systems combined with silver nanoparticles, demonstrated AgNPs connection with self-etching dentin bonding system may have a serious clinical impact [9]. Nagano F found that Colloidal Platinum Nanoparticles (CPN) can prolong the durability of bonds, creating a higher conversion at the interface [10]. As a new dental restorative material, new nano-hydroxyapatite composite resin has superior bonding ability and can be used in clinical therapy [7].

It is interesting that a sports drink containing $0.25 \%$ nano HA was effective in preventing dental erosion in situ [8]. Adding some nanomaterials into the common dental cure binder can cure dentine hypersensitivity by blocking off dentin tubules [11].

In an antibacterial aspect, the AgNPs and drug blended AgNPs showed a significant antibacterial and antifungal activity [9]. And composite resins containing silver or zinc oxide nanoparticles exhibited antibacterial activity against streptococcus mutans and lactobacillus [10]. The novel nano antibacterial inorganic filler, that contained a quaternary ammonium salt with long chain alkyl, showed a strong antibacterial property. It also exhibited good compatibility with the dental resin matrix after undergoing coupling treatment [12].

Nanomaterials could be used in the dental implants. Pang's results indicated that the nano hydroxy apatite/ $\mathrm{ZrO}_{2}$ composite coating on the surface of titanium materials has good biological activity and compatibility [13]. The novel nano 58S BG might be a better potential candidate for dentin pulp complex regeneration and can induce the differentiation and mineralization of HDPCs more efficiently, compared with regular BG(bioactive glass) [14].

\section{Biosafety of Nanomaterials}

Nanoparticles (NPs), which are contained in a variety of products of daily use and in medical products, may cause cytotoxicity. Size, shape, surface charge, surface hydrophobicity and heavy metal content determine cytotoxicity [15]. Almost all non-biodegradable NPs cause cytotoxic effects but employ quite different modes of action. Generation of reactive oxygen and nitrogen species by NPs and of metal ions due to dissolution of the NPs is discussed as a cause for cytotoxicity [16]. With few exceptions, smaller NPs are more toxic than larger ones [17]. In light of toxicological research, nanosilver is not inert to the body. The inhalation of silver nanoparticles has an adverse effect mainly on the liver and lung of rats. The oxidative stress caused by reactive oxygen species is responsible for the toxicity of nanoparticles, contributing to cytotoxic and genotoxic effects. The activity of the readily oxidized nanosilver surface underlies the molecular mechanism of toxicity [18]. Recent studies have demonstrated that Nano $\mathrm{TiO}_{2}$ induces DNA damage and increased the risk of cancer and the mechanism might relate with oxidative stress [19]. Wang's results reveal that the cytotoxicity of AgNPs is largely due to the chemical transformation of particulate silver from elemental silver $(\operatorname{Ag}(0)) n$ to $\mathrm{Ag}(+)$ ions and Ag-O-, then Ag-S- species [20].

\section{The Role of Cell Autophagy in Nanomaterial Induced Toxicity}

\section{The overview of autophagy}

Autophagy is an highly conserved intracellular catabolic process in eukaryotes, which can for eliminate harmful components to maintain cellular homeostasis. It mainly contains a complex lysosomal degradative process involved in multiple membrane structures ranging from endoplasmic reticulum to autophagosome. Autophagy will be kept at a lower level when nutritionally adequate, which called basal autophagy, only redundant or damaged organelles will be eliminated. It can instant on if poor nutrition, synthesizing proteins, producting ATP, the crucial energy and inducing gluconeogenesis. When the level of autophagy is sostenuto rising and can't be restrained, mass degradation of cell components will greet apoptosis. In recent years the range of autophagy substrates has also been extended to lipids termed lipophagy. Autophagy is recognized as playing a central role in cell survival and longevity.

Mammalian cells use three basic autophagic pathways for "self eating": macroautophagy, microautophagy and Chaperone Mediated Autophagy (CMA) [21].

All of them use lysosomes as a tool for degradation of substrates. They differ from each other in mechanism by which cargo is delivered to the lysosomes and each of them is also activated under different conditions. Processes of microautophagy and macroautophagy include participation of membrane. During macroautophagy, a new double membrane vesicle (autophagosome) is created and surrounds the targeted cytosolic area. Microautophagy is then mediated by lysosomal membrane itself and cytosolic content is engulfed by lysosomal membrane directly. Opposed to the micro and macroautophagy, CMA is based on direct translocation of substrate across the lysosome membrane via special proteins called chaperones. CMA is a highly selective process which targets only cytosolic proteins. There are two major steps in CMA; firstly, proteins determined to degradation are recognized and secondly, they are delivered through the membrane of lysosome. Chaperone Hsc70 with co chaperones recognizes a specific peptide sequence of protein. Then protein/chaperone complex interacts with lysosome associated membrane protein type 2A (LAMP-2A) which is located on the cytosolic side of lysosomal membrane. The full unfolding of the protein is required in order to cross through the lysosomal membrane [22].

More than 30 Autophagy related genes (Atgs) have been discovered in yeast and play important roles in autophagy, many of which have mammalian homologs. Many molecules are involved in the formation and working process of autophagy. Atg8/LC3, Atg7 and Atg6/ Beclin-1 with the class III phosphoinositide 3-kinase (PI3K) complex are the best characterized. The process of autophagosome formation involves three major steps: 1) Uncoordinated 51-Like Kinase (ULK) 1 helps in the double membrane formation in the initiation phase, 2) nucleation with the Beclin-1/class III PI3K complex and 3) elongation of the isolation membrane with the help of LC3 lipidationConjugation of yeast Atg8 or mammalian LC3 with Phosphatidylethanolamine (PE) during autophagy results in the formation of an insoluble form of Atg8 (Atg8-PE) or LC3 (LC3-II) which contributes to autophagosome formation. Atg8/LC3 stays on the membrane until it is degraded by lysosomes.

Therefore, Atg8/LC3 is widely used as a marker for monitoring autophagy. Several signaling pathways are involved in the regulation of 
autophagy, among which, the class I PI3K/Akt/mTOR signaling pathway is the most classic one. Autophagy is a self degradation process to recycle cellular components under stressed conditions, such as a lack of nutrients. Autophagy has been regarded as a nonselective process in the past however, accumulating evidence shows that it can be selective to remove specific proteins and damaged organelles, such as mitochondria under certain conditions [21]. Autophagy consists of 4 sequential steps: initiation of autophagosome formation, elongation and closure of autophagic membrane, fusion between autophagosome and lysome and degradation. In many cellular cases, autophagic induction is regulated by the mammalian target of rapamycin (mTOR) and the AMP-Activated Protein Kinase (AMPK). mTOR inactivates unc-51like kinase $1 / 2$ (ULK1/2) by phosphorylation under basal conditions, but mTOR is inhibited under stress conditions, allowing ULK1/2 to be modified to their active forms by AMPK phosphorylation initiating autophagy. Next, VPS34 lipid kinase complex and phosphatidylinositol 3-phosphate (PI3P) are recruited to complete autophagosome formation. In the step of elongation and closure of the autophagic membrane, the ubiquitin like proteins, ATG12 and ATG5 are conjugated and then the conjugated forms an E3-like ligase complex with ATG16L1. After that, ATG8 (LC3 and GABARAP subfamilies) is conjugated to the lipid phosphatidylethanolamine by the ATG16L1 ligase complex and in the case of LC3, LC3-I form changes to LC3-II form, which is often used as an autophagosome marker. VPS34-Beclin 1 complex containing Ultraviolet Radiation resistance Associated Gene protein (UVRAG) is involved in the step of fusion between the autophagosome and lysosome. Once the autophagosome and lysosome are fused to the autolysosome, the cargo in the autolysosome is degraded through lysosomal hydrolase activity. When there are certain proteins or organelles to be eliminated, they are ubiquitinated by E3 ubiquitin ligases and the ubiquitin chains serve as 'eat-me' signals. Then, adaptor proteins, such as $\mathrm{p} 62$ and NBR1, which contain a ubiquitin-associated UBA domain and LC3-Interacting Region (LIR), capture the ubiquitinated cargos and recruit LC3-II to proceed to selective autophagy. Autophagy is a like a double edged sword for a cell. On one hand, the amino acids created by autophagy can synthesize proteins and maintain the level of ATP, which can support cell survival in response to starvation or stress. Autophagy as well as other lysosome dependent systems, also functions in the degradation of dysfunctional proteins. In addition to promoting basal protein and organelle turnover, autophagy manifests cytoprotective effects through the regulation of catabolic processes and the clearance of pathogens. On the other hand, autophagy may induce apoptosis due to excessive essential organelle consumption. A correlation between autophagy and autoimmune disease has recently been confirmed. Autophagy has emerged as a mediator in the pathogenesis of RA [23]. The dual fuction of autophagy is also embodied in cancer sites it removes damaged organelles to avoid the accumulation of oxygen free radicals and mutation, but limit cell necrosis and the spread of inflammation, helping tumor cells survive in metabolic stress and immune suppression at the same time.

In addition to the above, autophagy can solve therapy resistance and tumor relapse problems [24], take place in the heat shock response as well [25]. Autophagy is increased in many long lived model organisms and contributes significantly to their longevity [26]. Understanding the molecular regulation of macroautophagy in relation to aging may offer new avenues for the treatment of age related diseases.

\section{Autophagy and nanomaterials}

Mechanism and expression: Take the CNS for instance, NPs can be potentially hazardous in terms of nano neurotoxicity via several possible mechanisms such as oxidative stress, autophagy and lysosome dysfunction and the activation of certain signaling pathways. Significant glutathione depletion and reactive oxygen species generation, which reduced the cellular antioxidant level and caused cytoskeleton disruption.

A growing body of evidence suggests that autophagy is activated upon internalization of engineered nanomaterials, most likely as a protective response to what is perceived as foreign or toxic. We find that nanoparticles within a certain size range acts as potent autophagy activator and that autophagic flux is an underlying physiological process of the cellular clearance of the nanoparticles. Park's [27] research shows that SWCNTs induce autophagic cell death through mitochondrial dysfunction and cytosolic damage in human bronchial epithelial cells. At $24 \mathrm{~h}$ after exposure, SWCNTs rapidly decreased ATP production and cell viability as well a slight increase in the number of cells in the subG1 and G1 phases. In addition, SWCNTs increased the expression of superoxide dismutase SOD-1, but not SOD-2 and the number of cells generating ROS. The concentration of $\mathrm{Cu}$ and $\mathrm{Zn}$ ions and the release of nitric oxide, interleukin (IL)-6, and IL-8 also increased in a dose dependent manner in cells exposed to SWCNTs. Furthermore, the levels of autophagy related genes, especially the DRAM1 gene, and the autophagosome formation related proteins, were clearly up regulated together with an increase of autophagosome like vacuoles. GNP has been proven to have a similar effect, caused mitochondria damage and elevated the levels of autophagy related proteins [28]. Functionalized Single Walled carbon Nanotubes (SWNT) restored normal autophagic activity by reversing abnormal activation of mTOR signaling and deficits in lysosomal proteolysis, thereby facilitating elimination of autophagic substrates [29]. As nano carbon MWCNTs could induce cognitive deficits, histopathological alteration and changes of autophagy levels (increased the ratio of LC3 II /LC3 I and the expression of Beclin-1) [30]. However, degradation of the autophagic substrate p62 protein was also inhibited by both nanomaterials [31]. Chen's researches demonstrated that GO treatment of cells simultaneously triggers autophagy and TLR4/TLR9-regulated inflammatory responses and the autophagy was at least partly regulated by the TLRs pathway [32].

The experiments related to silicon dioxide usually aim at endothelial cells. Duan and his team found that SiNPs induce autophagic activity in endothelial cells and pericytes, subsequently disturb the endothelial cell homeostasis and impair angiogenesis. The VEGFR2 mediated autophagy pathway may play a critical role in maintaining endothelium and vascular homeostasis [33]. The typically morphological characteristics (autophagosomes and autolysosomes) of the autophagy process were observed in TEM ultra structural analysis by Yu's team [34]. Their findings demonstrated that the SNPs induced autophagy and autophagic cell death was triggered by the ROS generation in HepG2 cells, which resulted in a dose dependent manner. Duan's findings demonstrated that $\mathrm{Nano}_{\mathrm{SiO}}$ could disturb the $\mathrm{NO} /$ NOS system, induce inflammatory response, activate autophagy, and eventually lead to endothelial dysfunction via the PI3K/Akt/mT or pathway [35]. Ha's findings further revealed that NP1 stimulates autophagy including the processing of LC3 $\beta$ I to LC3 $\beta$ II, a key protein involved in autophagosome formation, which is dependent on ERK1/2 signaling [36]. Schütz's research showed us that internalized SiNPs accumulate in lysosomes resulting in inhibition of autophagy mediated protein turnover and impaired degradation of internalized epidermal growth factor, whereas endosomal recycling proceeds unperturbed [37]. 
In addition of nano carbon and nano silicon dioxide, many NPS were demonstrated the ability to induce autophagy. AgNPs induced autophagic flux defect may consequently lead to aggravated cytotoxic responses [38]. Park's results showed us that M FeNPs induce autophagy preceding apoptosis through mitochondrial dysfunction and ER stress in RAW 264.7 cells [39]. The mechanism is similar to carbon nano as we said above. The uptake of $\mathrm{TiO}_{2}$ NPs led to a dose dependent increase in autophagic effect under non cytotoxic conditions, which has been demonstrated in Lopes's research [40]. Leaching of $\mathrm{Cu}$ ions, reactive oxygen species generation and autophagy appear to be the underlying mechanisms of $\mathrm{Cu}$ NP toxicity in lung cells [41]. Lu proposes that Lipid Nanoparticles (LNs) induce autophagy lysosome signaling and neurovascular response at least partially via an Atg5 dependent pathway [42]. In Jawaid's study, it is said that the effect of platinum nanoparticles on cell death were induced by ultrasounds in human lymphoma U937 cells. It is the first study to suggest autophagy as a pro survival pathway via ultrasound. The combination of Pt NPs and ultrasound might be a fantastic alternative in cancer treatment [43]. While exposure to NiNPs suppresses the methionine repairing enzymatic MSRA and MSRB3 production, which could lead to the dysfunction of autophagy and ERK phosphorylation [44]. PAMAM nano particles promote injury by inducing autophagic cell death through the Akt-TSC2-mTOR signaling pathway [45]. And for Inorganic nano materials, it has been thought to induce autophagy, maintaining the cellular homeostasis [46]. Wang Y first showed us that AANTs are nontoxic and can activate autophagy in different cell types, which provides us with more opportunities for future clinical cancer therapy [47].

Influence factor: The chemical and physical properties of a nanometer material itself has a decisive effect on its toxicity, including size, shape, surface charge, surface finish, metallic impurity, decentrality, degradability and the formation of protein corona. The degree of absorption in the cell is dependent on the characteristics of the nanoparticles. Exposed pathways also have an effect. Andón found that acute exposure of cells to Carbon Nanotubes (CNTs) can induce apoptosis whereas chronic exposure to CNTs may yield an apoptosis resistant and tumorigenic phenotype in lung epithelial cells [48]. Various univariate experiments designed by Huang demonstrate that nanomaterials induce autophagy in a dispersity dependent manner [49].

\section{Common Dental Nano Materials Impact on Autoph-} agy

\section{Nano composite resins}

Nowadays, nano composite resins are some of the most important and latest in oral application materials. On one hand, nano composite resin can be used with other materials such as carbon nanoparticles and zinc oxide nanoparticles. It improved the overall performance of composite resins, especially in improving abrasion resistance and decreasing polymerization shrinkage [50].

On the other hand, nano composite resins can also be used alone. As a new dental restorative material, new nano hydroxyapatite composite resin has superior bonding ability and can be used in clinical therapy [12].

\section{Carbon nanoparticles}

Carbon nanoparticles have shown potential applications in many fields, added indental resin based composites [51], serving as adhesive material or wear protective coatings for dental implants, artificial joints, etc [52]. But it is a double edged sword. Many researches on its potential harm and its mechanism have been done.

Recently, Orecna pointed that carboxylated Multi Walled Carbon Nanotubes (MWCNTs) induce a decrease in viability of cultured $\mathrm{Hu}-$ man Umbilical Vein Endothelial Cells (HUVECs) associated with the profound accumulation of autophagosomes. They investigated the mechanisms of toxicity of multi walled carbon nanotubes on human endothelial cells, concluding the idea that pharmacological stimulation of autophagic flux may represent a new method of cytoprotection against the toxic effects of these nanomaterials [53].

There is also a study performed on normal mouse lung, the aim of Yu's study was to investigate the potential toxic mechanisms associated with Multiwall Carbon Nanotubes (MWCNT) in normal mouse lung. The results indicated that PMWCNT induced pulmonary autophagy accumulation and resulted in more potent tumorigenic effects compared to TMWCNT [54].

Several studies have reported that Multi Walled Carbon Nanotubes (MWCNTs) induce apoptosis and oxidative damage on hippocampal synaptic plasticity and spatial cognition in rats in vitro experiments and could induce cognitive deficits in vivo via the increased autophagic levels [30]. Bramini's research showed that although graphene exposure does not impact neuron viability, it does nevertheless have important effects on neuronal transmission and network functionality, thus warranting caution when planning to employ this material for neurobiological applications [55].

Also, it is demonstrated that MWCNT cell interactions can be modulated by varying densely distributed surface ligands on MWCNTs. Using a fluorescent autophagy reporting cell line, the researchers evaluated the autophagy induction capability of 81 surface-modified MWCNTs. They identified strong and moderate autophagy-inducing MWCNTs as well as those that did not induce autophagy. Variation of the surface ligand structure of strong autophagy nanoinducers led to the induction of different autophagy activating signaling pathways, presumably through their different interactions with cell surface receptors [56] .

However, it is a different story when Bronchial epithelial cells and mesothelial cells becomes crucial targets for the safety assessment of inhalation of Carbon Nanotubes (CNTs), which resemble asbestos particles in shape. The researchers verified that MWCNTs are actively internalized into HBECs and HMCs and were accumulated in the lysosomes of the cells after 24 hour treatment. Next, they determined which endocytosis pathways (clathrin mediated, caveolae mediated, and macropinocytosis) were associated with MWCNT internalization by using corresponding endocytosis inhibitors, in two nonphagocytic cell lines derived from bronchial epithelial cells and mesothelioma cells. Clathrin mediated endocytosis inhibitors significantly suppressed MWCNT uptake, whereas caveolae mediated endocytosis and macropinocytosis were also found to be involved in MWCNT uptake. Thus, MWCNTs were positively taken up by nonphagocytic cells, and their cytotoxicity was closely related to these three endocytosis pathways [57].

Chen GY demonstrated that GO treatment of cells simultaneously triggers autophagy and TLR4/TLR9-regulated inflammatory responses, and the autophagy was at least partly regulated by the TLRs pathway, suggesting a mechanism by which cells respond to nanomaterials and underscores the importance of future safety evaluation of nanomaterials [32]. 
Single walled carbon nanotubes and graphene oxides, and found that both carbon nanomaterials induced adverse effects in murine peritoneal macrophages, and GOs were more potent than $\mathrm{AF}$ SWCNTs. Both carbon nanomaterials induced autophagosome accumulation and the conversion of LC3 I to LC3 II. However, degradation of the autophagic substrate $\mathrm{p} 62$ protein was also inhibited by both nanomaterials. Further analyses on lysosomes revealed that both carbon nanomaterials accumulated in macrophage lysosomes, leading to lysosome membrane destabilization, which indicates reduced autophagic degradation [31]. The nanoparticles can work on autophagosome accumulation and genes.

Tsukahara's study suggests that the levels of autophagy related genes (LC3B) and autophagosome formation are clearly up regulated, along with an increase in numbers of autophagosome vacuoles. His review highlights the importance of autophagy as an emerging mechanism of CNT toxicity [58].

\section{Nano $\mathrm{TiO}_{2}$}

Nano titanium dioxide $\left(\mathrm{NP} \mathrm{TiO}_{2}\right)$ is widely used in various fields, including sun cream, cosmetics and so on. In oral terms, Nano titanium dioxide is a promising candidate for orthopedic and dental implants, which can reinforce the physical mechanical properties of dental resin composites [59]. It is also a promising material for restoration because of its potential antibacterial activity and durable restoration to withstand forces exerted via mastication [60]. And analogously, it selectively enhances soft tissue integration and inhibits bacterial reproduction [61]. However, with the development of the application of $\mathrm{TiO}_{2}$ nano titanium dioxide, more and more attention to their toxicity to humans is also increasing. Many studies have reported that NP $\mathrm{TiO}_{2}$ can cause a variety of cell toxicity.

For example, Kyeong Nam Yu et al., proposed that to determine if autophagy was induced in $\mathrm{TiO}_{2} \mathrm{NP}$ treated cells, the expression levels of LC3, sequestosome-1 (SQSTM1/p62), and BECN1 were examined by western blot assay and densitometric analysis. LC3 is one of the key regulators of autophagy, which is measured quantitatively by the ratio of LC3 II to LC3 I. The results clearly demonstrate that the LC3 II/LC3 I ratio increased significantly in the $\mathrm{TiO}_{2} \mathrm{NP}$ treated cells and the $\mathrm{TiO}_{2}$ NP induces autophagy in human bronchial epithelial cells [62]. In addition, WenlinBai reported that over expressed miR34a enhanced TNP induced autophagy and cell death through targeted down regulation of Bcl 2 in BEAS 2B cells [50]. Another study by Shi et al. provides evidence that $\mathrm{TiO}_{2} \mathrm{NPs}(5-20 \mathrm{~nm})$ can penetrate the skin and interact with the immune system [63]. In addition, the presence of $14 \mathrm{~nm}$ silica coated $\mathrm{TiO}_{2} \mathrm{NPs}$ within the epidermis and superficial dermis has been observed [64]. Viviana's findings provided evidence of the autophagic effects by $\mathrm{TiO}_{2}$ NPs in a dose-dependent manner and under non cytotoxic conditions. Depending on the initial dose, $\mathrm{TiO}_{2} \mathrm{NPs}$ can switch the autophagic response of keratinocytes between blockage and induction. $\mathrm{TiO}_{2} \mathrm{NPs}$ mediated autophagy induction at low dose can be interpreted as a standard mechanism by which cells attempt to increase the clearance of NPs. While the halt in autophagy at high dose may indicate that the cellular response cannot cope with a NPs overload and thus show a deficient degradative capacity [40].

Other studies showed that $\mathrm{TiO}_{2}$ NPs could induce autophagy in normal lung cells and in primary human keratinocytes [65]. Based on the studies illustrated above, we hypothesize that $\mathrm{TiO}_{2} \mathrm{NPs}$ can induce autophagy dysfunctions in brain tissues and cells. Therefore, autophagy could be a potential mechanism underlying $\mathrm{TiO}_{2} \mathrm{NPs}_{\text {induced neu- }}$ rotoxicity. However, more studies are needed to further investigate the relationship between brain damage and $\mathrm{TiO}_{2} \mathrm{NPs}$ mediated autophagy dysfunction [66]. Some findings indicate that nano $\mathrm{TiO}_{2}$ induces autophagy through activating AMPK to inhibit mTOR in podocytes, and such autophagy plays a protecting role against oxidative stress on the cell proliferation [67]. We used a whole body chamber inhalation system to expose $\mathrm{A} / \mathrm{J}$ mice to $\mathrm{TiO}_{2}$ nanoparticles for 28 days. During the experiments, the inhaled $\mathrm{TiO}_{2}$ nanoparticles were characterized using a cascade impactor and transmission electron microscopy. After inhalation of the $\mathrm{TiO}_{2}$ nanoparticles, hyperplasia and inflammation were observed in a $\mathrm{TiO}_{2}$ dose dependent manner. To determine the biological mechanism of the toxic response in the lung, we examined Endoplasmic Reticulum (ER) and mitochondria in lung. The ER and mitochondria were disrupted and dysfunctional in the $\mathrm{TiO}_{2}$ exposed lung leading to abnormal autophagy [68]. This showed us that NPs are internalized by human brain derived endothelial cells; however, the extent of their intracellular uptake is dependent on the characteristics of the NPs. After their uptake by human brain derived endothelial cells NPs are transported into the lysosomes of these cells, where they enhance the activation of lysosomal proteases. In brain derived endothelial cells, NPs induce the production of an oxidative stress after exposure to iron oxide and $\mathrm{TiO}_{2} \mathrm{NPs}$, which is correlated with an increase in DNA strand breaks and defensive mechanisms that ultimately induce an autophagy process in the cells [69] .

$\mathrm{TiO}_{2}$ NPs possess unique characteristics due to their tiny size and are widely used in many fields, but the toxic effects on cells are not negligible. Future research will be more focused on the effects of nanomaterials on autophagy.

\section{Nano Ag}

Nano silver is widely used in oral medicine as a high biological safety and not easy to induce the resistance of bacteria. Such as antimicrobial resin material and so on.

Ying Lee Tzu and his team collected blood and liver tissue after exposure to $1,4,7,10$ and 30 days, respectively, by intraperitoneal injection of $500 \mathrm{~kg} 1 \mathrm{Ag}$ concentration of NPs $\mathrm{mg}$ in rats [42]. The silver nanoparticles absorbed by the liver were very rapid, and were mainly found in the lysosome. At different time points of injection of silver nano particles, serum AST and ALT in enzyme activity are different, and showed an inflammatory response in about 4 days after injected with $500 \mathrm{mg} \mathrm{kg} ~(-1)$ concentration of Ag NPs. The reaction in 7-10 days increased modestly, and the inflammatory response reaches the top on $30^{\text {th }}$ day. At the same time, according to the TEM, they found that LC3 II's aggregation and LC3 II protein expression obtained under certain silver nanoparticles concentration, early autophagy is induced, and showed a time dependent, decreased gradually, and ATP may play a significant role on induction of liver toxicity after administered silver nanoparticles.

On the other hand, Lin J and his team also had done some researches for silver nanoparticles [70]. They use HeLa EGFP LC3 to detect autophagy. By transmission electron microscope showed that silver nanoparticles on HeLa cells have more autophagosomes than the cells which are not to be treated. At the same time, high magnification of the image displayed, autophagic vacuoles containing partial degradation of cytoplasmic material performance out of the increase in the electron density. It is concluded that the silver nanoparticles can induce the accumulation of autophagosomes. Subsequently, they even did more experiments to get the following conclusions; silver nanoparticles enhanced the formation of autophagy and induced autophagy by Ptdins $3 \mathrm{k}$ dependent and MTOR independent methods but 
it did not destroy the lysosomal function. And due to the release of silver ions, silver nanoparticles did not cause significant cytotoxicity. Silver nanoparticles will play a protective role when induced in autophagy, and inhibits the autophagy by enhancing the cell's cytotoxicity by chemical inhibitors or ATG5 [38]. AgNPs also have been shown to interfere with ubiquitin modifications, either via up regulating levels of enzymes participating in ubiquitination, or through impairing the biological reactivity of ubiquitin. Ubiquitination both confers selectivity to autophagy as well as modulates stabilization, activation, and trafficking of proteins involved in autophagic clearance pathways.

\section{Nano $\mathrm{SiO}_{2}$}

Compared with other nanoparticles, $\mathrm{Nano} \mathrm{SiO}_{2}$ are one of the most widely used nanomaterials due to their favorable optical properties, high hydrophilicity, large surface area, fantastic modification and good biocompatibility. And it also has a wide range of applications in the field of oral medicine, such as maxillofacial prostheses, in which area nano $\mathrm{SiO}_{2}$ can effect some mechanical properties of maxillofacial silicone elastomer [71].

Although nano silver may have great potential for biomedical applications, there is still a lack of a correlative safety evaluation on the cardiovascular system. So, Duan J and his team had done some research on that [35]. Their study was aimed to clarify the biological behavior and influence of $\mathrm{Nano}_{\mathrm{SiO}}$ on endothelial cell function. Nano $\mathrm{SiO}_{2}$ could induce the depolarization of mitochondria and lead to mitochondrial damage. Nano $\mathrm{SiO}_{2}$ induced mitochondrial damage had a close connection with autophagy. The damaged mitochondria can be removed by mitophagy (mitochondria specific autophagy). Their findings demonstrated that $\mathrm{Nano} \mathrm{SiO}_{2}$ can induce autophagic activity, accompanied by NO/NOS system disorder and an inflammatory response, eventually leading to endothelial cell dysfunction via the inhibition of the PI3K/Akt/mTOR signaling pathway. Their findings also confirm that $\mathrm{Nano} \mathrm{SiO}_{2}$ induced endothelial cell dysfunction is associated with autophagy, which may be an important mechanism and adverse outcome pathway of cardiovascular diseases caused by nanoparticles [33]. JunchaoDuan and his team found that the cardiovascular toxicity triggered by SiNPs occurs mainly in vascular endothelium rather than cardiomyocytes. The SiNPs can disrupt the cytoskeleton organization, activate autophagic activity in endothelial cells and percytes, cause mitochondria damage, and attenuate the expression of cellular adhesion molecules, which contribute to the disturbance of the endothelial cell homeostasis, and eventually impair angiogenesis. VEGFR2/PI3K/Akt/mTOR and VEGFR2/MAPK/Erk1/2/mTOR signaling pathway are involved in SiNPs induced cardiovascular toxicity. Moreover, there is a crosstalk between the VEGFR2 mediated autophagy signaling and angiogenesis signaling pathways [35]. In addition, researchers have found that the stimulation of autophagy and associated signaling suggests a cellular mechanism for the stimulatory effects of silica nanoparticles on osteoblast differentiation and mineralization.

\section{Nano ZnO}

The increasing risk of incidental exposure to nanomaterials has led to mounting concerns regarding nanotoxicity.

As zinc oxide nanoparticles ( $\mathrm{ZnO} \mathrm{np}$ ) are used in an increasing number of industrial products such as paint, coating and cosmetics. As $\mathrm{ZnO} n p$ display antimicrobial activity and reduce growth of bacterial biofilms, they are commonly blended in the resin based dental composites for restoration [72]. Assessing the toxicity mechanism in normal skin cells seems not dispensable. So Yu started a research, which finally gave a conclusion that Zinc oxide nanoparticles induced autophagic cell death and mitochondrial damage via reactive oxygen species generation [73].

Also, Zinc oxide nanoparticles ( $\mathrm{ZnO} \mathrm{NPs}$ ) have raised serious concerns about their impact on the health and environment for wide biological applications. Pati investigated genotoxic, clastogenic and cytotoxic effects of $\mathrm{ZnO}$ NPs on macrophages and in adult mice. Studies revealed that administration of higher dose caused severe DNA damage and inhibited DNA repair mechanism by down regulating the expression offen 1 and polB proteins. Finally they concluded that $\mathrm{ZnO}$ np leads to cell death through cell autophagy and mitochondria damage [74].

Johnson told us that $\mathrm{ZnO}$ NPs are immunotoxic to primary and immortalized immune cells in his research. ZnO NP mediated immune cell death was associated with increased levels of intracellular Reactive Oxygen Species (ROS). It was not apoptosis, necroptosis or pyroptosis that was due to $\mathrm{ZnO}$ NP death [71]. While Roy told us a different story. His study demonstrates autophagy supports apoptosis on $\mathrm{ZnO}$ NPs exposure. They believed that it is depleting antioxidant enzymes, increasing lipid peroxidation and protein carbonyl contents in macrophages $\mathrm{ZnO}$ NPs induced ROS generation promoting autophagy in cells [75].

\section{Reduce autophagy}

Some researches show that Thapsigargin (TG) can inhibited autophagy. Wang $\mathrm{C}$ and his team had done some research about TG. TG, is widely used to induce endoplasmic reticular stress. TG induced autophagy can be confirmed by the accumulation of MDC, GFP LC3 staining autophagic vacuoles, and the improved expression of LC3 II and Beclin 1. Additionally, chloroquine can markedly inhibited autophagy.

\section{Summary and Outlook}

Many nano materials have been confirmed that induce cell autophagy. However, the mechanism of NM nanotoxicity and cause of protective autophagy is still not clear. In addition, the relationship between NPs induced autophagy and other inherent effect as well as the application in tumor treatment still need further study. A lack of common standards is one of the limitations of the current studies of NP cytotoxicity. Different cell lines, exposure times and colorimetric assays are used in different studies, which make it difficult or even impossible to compare cytotoxic effect among these results. A new generation of nano materials worth looking forward to, which has better biocompatibility, reduces oxidative stress effect and focuses on biosafety.

\section{Acknowledgment}

This work was supported by the Zhejiang Provincial Natural Science Foundation of China (LY16H140003, LY17H140008), the Wenzhou Municipal Science and Technology Bureau Foundation of China (Y20150070).

\section{Conflicts of Interest}

The authors declare no conflict of interest. 


\section{References}

1. Bleeker EA, de Jong WH, Geertsma RE, Groenewold M, Heugens EH, et al. (2013) Considerations on the EU definition of a nanomaterial: science to support policy making. Regul Toxicol Pharmacol 65: 119-125.

2. Junling W, Qiang Z, Ruinan S, Ting Z, Jianhua G, et al. (2015) [Dental plaque microcosm biofilm behavior on a resin composite incorporated with nano-antibacterial inorganic filler containing long-chain alkyl quaternary ammonium salt]. Hua Xi Kou Qiang Yi Xue Za Zhi 33: 565-569.

3. Chiang YC, Lin HP, Chang HH, Cheng YW, Tang HY, et al. (2014) A mesoporous silica biomaterial for dental biomimetic crystallization. ACS Nano 8 : 12502-12513.

4. Lu X, Xia Y, Liu M, Qian Y, Zhou X, et al. (2012) Improved performance of diatomite-based dental nanocomposite ceramics using layer-by-layer assembly. Int J Nanomedicine 7: 2153-2164.

5. Atai M, Pahlavan A, Moin N (2012) Nano-porous thermally sintered nano silica as novel fillers for dental composites. Dent Mater 28: 133-145.

6. Swarup SJ, Rao A, Boaz K, Srikant N, Shenoy R (2014) Pulpal response to nano hydroxyapatite, mineral trioxide aggregate and calcium hydroxide when used as a direct pulp capping agent: an in vivo study. J Clin Pediatr Dent 38 201-206.

7. Zhao MM, Wang QS, Wang S, Wang FH, Shu JY (2013) [Bonding performance evaluation between the new nano-hydroxyapatite composite resin and dentin]. Shanghai Kou Qiang Yi Xue 22: 274-277.

8. Min JH, Kwon HK, Kim BI (2015) Prevention of dental erosion of a sports drink by nano-sized hydroxyapatite in situ study. Int J Paediatr Dent 25: 61-69.

9. Emmanuel R, Palanisamy S, Chen SM, Chelladurai K, Padmavathy S, et al. (2015) Antimicrobial efficacy of green synthesized drug blended silver nanoparticles against dental caries and periodontal disease causing microorganisms. Mater Sci Eng C Mater Biol Appl 56: 374-379.

10. Kasraei S, Sami L, Hendi S, AliKhani M-Y, Rezaei-Soufi L, et al. (2014) Antibacterial properties of composite resins incorporating silver and zinc oxide nanoparticles on Streptococcus mutans and Lactobacillus. Restor Dent Endod 39: 109-114.

11. Taha ST, Han H, Chang SR, Sovadinova I, Kuroda K, et al. (2015) Nano/ micro fluorhydroxyapatite crystal pastes in the treatment of dentin hypersensitivity: an in vitro study. Clin Oral Investig 19: 1921-1930.

12. Junling W, Kaiyun Z, Ting Z, Chuanjian Z (2014) [Synthesis of a nano-antibacterial inorganic filler containing a quaternary ammonium salt with long chain alkyl and its effect on dental resin composites]. Hua Xi Kou Qiang Yi Xue Za Zhi 32: 513-518.

13. Pang $X$, Huang $Y(2012)$ Physical properties of nano-HAs/ZrO2 coating on surface of titanium materials used in dental-implants and its biological compatibility. J Nanosci Nanotechnol 12: 902-910.

14. Gong W, Huang Z, Dong Y, Gan Y, Li S, et al. (2014) lonic extraction of a novel nano-sized bioactive glass enhances differentiation and mineralization of human dental pulp cells. J Endod 40: 83-88.

15. Meindl C, Kueznik T, Bösch M, Roblegg E, Fröhlich E (2015) Intracellula calcium levels as screening tool for nanoparticle toxicity. J Appl Toxicol 35: 1150-1159.

16. Fröhlich E (2013) Cellular targets and mechanisms in the cytotoxic action of non-biodegradable engineered nanoparticles. Curr Drug Metab 14: 976-988.

17. Shang L, Nienhaus K, Nienhaus GU (2014) Engineered nanoparticles interacting with cells: size matters. J Nanobiotechnology 12: 5.

18. Świdwińska-Gajewska AM, Czerczak S (2015) [Nanosilver--Occupational exposure limits]. Med Pr 66: 429-442.

19. Shi Z, Niu Y, Wang Q, Shi L, Guo H, et al. (2015) Reduction of DNA damage induced by titanium dioxide nanoparticles through Nrf2 in vitro and in vivo. J Hazard Mater 298: 310-319.
20. Wang L, Zhang T, Li P, Huang W, Tang J, et al. (2015) Use of Synchrotron Radiation-Analytical Techniques To Reveal Chemical Origin of Silver-Nanoparticle Cytotoxicity. ACS Nano 9: 6532-6547.

21. Yang Z, Goronzy JJ, Weyand CM (2015) Autophagy in autoimmune disease. J Mol Med (Berl) 93: 707-717

22. Ondrej M, Cechakova L, Durisova K, Pejchal J, Tichy A (2016) To live or let die: Unclear task of autophagy in the radiosensitization battle. Radiother Oncol 119: 265-275.

23. Dai Y, Hu S (2016) Recent insights into the role of autophagy in the pathogenesis of rheumatoid arthritis. Rheumatology (Oxford) 55: 403-410.

24. Villar VH, Merhi F, Djavaheri-Mergny M, Durán RV (2015) Glutaminolysis and autophagy in cancer. Autophagy 11: 1198-1208.

25. Dokladny K, Myers OB, Moseley PL (2015) Heat shock response and autophagy--cooperation and control. Autophagy 11: 200-213.

26. Lapierre LR, Kumsta C, Sandri M, Ballabio A, Hansen M (2015) Transcriptional and epigenetic regulation of autophagy in aging. Autophagy 11: 867-880.

27. Park EJ, Zahari NE, Lee EW, Song J, Lee JH, et al. (2014) SWCNTs induced autophagic cell death in human bronchial epithelial cells. Toxicol In Vitro 28 : $442-450$.

28. Park EJ, Lee GH, Han BS, Lee BS, Lee S, et al. (2015) Toxic response of graphene nanoplatelets in vivo and in vitro. Arch Toxicol 89: 1557-1568.

29. Xue X, Wang LR, Sato Y, Jiang Y, Berg M, et al. (2014) Single-walled carbon nanotubes alleviate autophagic/lysosomal defects in primary glia from a mouse model of Alzheimer's disease. Nano Lett 14: 5110-5117.

30. Gao J, Zhang X, Yu M, Ren G, Yang Z (2015) Cognitive deficits induced by multi-walled carbon nanotubes via the autophagic pathway. Toxicology 337 $21-29$

31. Wan B, Wang ZX, Lv QY, Dong PX, Zhao LX, et al. (2013) Single-walled carbon nanotubes and graphene oxides induce autophagosome accumulation and lysosome impairment in primarily cultured murine peritoneal macrophages. Toxicol Lett 221: 118-127.

32. Chen GY, Yang HJ, Lu CH, Chao YC, Hwang SM, et al. (2012) Simultaneous induction of autophagy and toll-like receptor signaling pathways by graphene oxide. Biomaterials 33: 6559-6569.

33. Duan J, Yu Y, Yu Y, Li Y, Huang P, et al. (2014) Silica nanoparticles enhance autophagic activity, disturb endothelial cell homeostasis and impair angiogenesis. Part Fibre Toxicol 11: 50

34. Yu Y, Duan J, Yu Y, Li Y, Liu X, et al. (2014) Silica nanoparticles induce autophagy and autophagic cell death in HepG2 cells triggered by reactive oxygen species. J Hazard Mater 270: 176-186.

35. Duan J, Yu Y, Yu Y, Li Y, Wang J, et al. (2014) Silica nanoparticles induce autophagy and endothelial dysfunction via the PI3K/Akt/mTOR signaling pathway. Int J Nanomedicine 9: 5131-5141.

36. Ha SW, Weitzmann MN, Beck GR Jr (2014) Bioactive silica nanoparticles promote osteoblast differentiation through stimulation of autophagy and direct association with LC3 and p62. ACS Nano 8: 5898-5910.

37. Schütz I, Lopez-Hernandez T, Gao Q, Puchkov D, Jabs S, et al. (2016) Lysosomal Dysfunction Caused by Cellular Accumulation of Silica Nanoparticles. J Biol Chem 291: 14170-14184.

38. Mao BH, Tsai JC, Chen CW, Yan SJ, Wang YJ (2016) Mechanisms of silver nanoparticle-induced toxicity and important role of autophagy. Nanotoxicology 10: 1021-1040.

39. Park EJ, Choi DH, Kim Y, Lee EW, Song J, et al. (2014) Magnetic iron oxide nanoparticles induce autophagy preceding apoptosis through mitochondrial damage and ER stress in RAW264.7 cells. Toxicol In Vitro 28: 1402-1412.

40. Lopes VR, Loitto V, Audinot JN, Bayat N, Gutleb AC, et al. (2016) Dose-dependent autophagic effect of titanium dioxide nanoparticles in human $\mathrm{HaCaT}$ cells at non-cytotoxic levels. J Nanobiotechnology 14: 22. 
41. Ahamed M, Akhtar MJ, Alhadlaq HA, Alrokayan SA (2015) Assessment of the lung toxicity of copper oxide nanoparticles: current status. Nanomedicine (Lond) 10: 2365-2377.

42. Lu NN, Liu J, Tian Y, Liao MH, Wang H, et al. (2014) Atg5 deficit exaggerates the lysosome formation and cathepsin $B$ activation in mice brain after lipid nanoparticles injection. Nanomedicine 10: 1843-1852.

43. Jawaid P, Rehman MU, Hassan MA, Zhao QL, Li P, et al. (2016) Effect of platinum nanoparticles on cell death induced by ultrasound in human lymphoma U937 cells. Ultrason Sonochem 31: 206-215.

44. Feng PH, Huang YL, Chuang KJ, Chen KY, Lee KY, et al. (2015) Dysfunction of methionine sulfoxide reductases to repair damaged proteins by nicke nanoparticles. Chem Biol Interact 236: 82-89.

45. Li C, Liu H, Sun Y, Wang H, Guo F, et al. (2009) PAMAM nanoparticles promote acute lung injury by inducing autophagic cell death through the AktTSC2-mTOR signaling pathway. J Mol Cell Biol 1: 37-45

46. Zhou W, Miao Y, Zhang Y, Liu L, Lin J, et al. (2013) Induction of cyto-protective autophagy by paramontroseite VO2 nanocrystals. Nanotechnology 24 165102

47. Wang Y, Kaur G, Chen Y, Santos A, Losic D, et al. (2015) Bioinert Anodic Alumina Nanotubes for Targeting of Endoplasmic Reticulum Stress and Autophagic Signaling: A Combinatorial Nanotube-Based Drug Delivery System for Enhancing Cancer Therapy. ACS Appl Mater Interfaces 7: 27140-27151.

48. Andón FT, Fadeel B (2013) Programmed cell death: molecular mechanisms and implications for safety assessment of nanomaterials. Acc Chem Res 46: 733-742.

49. Huang D, Zhou H, Gao J (2015) Nanoparticles modulate autophagic effect in a dispersity-dependent manner. Sci Rep 5: 14361.

50. Bai W, Chen Y, Sun P, Gao A (2016) Downregulation of B-cell lymphoma/ leukemia-2 by overexpressed microRNA 34a enhanced titanium dioxide nanoparticle-induced autophagy in BEAS-2B cells. Int J Nanomedicine 11: 1959-1971.

51. Zhang F, Xia Y, Xu L, Gu N (2008) Surface modification and microstructure of single-walled carbon nanotubes for dental resin-based composites. J Biomed Mater Res B Appl Biomater 86: 90-97.

52. Penkov OV, Pukha VE, Starikova SL, Khadem M, Starikov VV, et al. (2016) Highly wear-resistant and biocompatible carbon nanocomposite coatings fo dental implants. Biomaterials 102: 130-136.

53. Orecna M, De Paoli SH, Janouskova O, Tegegn TZ, Filipova M, et al. (2014) Toxicity of carboxylated carbon nanotubes in endothelial cells is attenuated by stimulation of the autophagic flux with the release of nanomaterial in autophagic vesicles. Nanomedicine 10: 939-948

54. Yu KN, Kim JE, Seo HW, Chae C, Cho MH (2013) Differential toxic responses between pristine and functionalized multiwall nanotubes involve induction of autophagy accumulation in murine lung. J Toxicol Environ Health A 76 : 1282-1292.

55. Bramini M, Sacchetti S, Armirotti A, Rocchi A, Vázquez E, et al. (2016) Graphene Oxide Nanosheets Disrupt Lipid Composition, Ca(2+) Homeostasis, and Synaptic Transmission in Primary Cortical Neurons. ACS Nano 10: 7154-7171.

56. Wu L, Zhang Y, Zhang C, Cui X, Zhai S, et al. (2014) Tuning cell autophagy by diversifying carbon nanotube surface chemistry. ACS Nano 8: 2087-2099.

57. Maruyama K, Haniu H, Saito N, Matsuda Y, Tsukahara T, et al. (2015) Endocytosis of Multiwalled Carbon Nanotubes in Bronchial Epithelial and Mesothelial Cells. Biomed Res Int 2015: 793186

58. Tsukahara T, Matsuda $\mathrm{Y}$, Haniu $\mathrm{H}$ (2014) The role of autophagy as a mechanism of toxicity induced by multi-walled carbon nanotubes in human lung cells. Int J Mol Sci 16: 40-48.
59. Yang T, Qian S, Qiao Y, Liu X (2016) Cytocompatibility and antibacterial activity of titania nanotubes incorporated with gold nanoparticles. Colloids Surf B Biointerfaces 145: 597-606.

60. Garcia-Contreras R, Scougall-Vilchis RJ, Contreras-Bulnes R, Sakagami H Morales-Luckie RA, et al. (2015) Mechanical, antibacterial and bond strength properties of nano-titanium-enriched glass ionomer cement. J Appl Oral Sci 23: $321-328$

61. Wang X, Lu T, Wen J, Xu L, Zeng D, et al. (2016) Selective responses of human gingival fibroblasts and bacteria on carbon fiber reinforced polyetheretherketone with multilevel nanostructured TiO2. Biomaterials 83: 207218.

62. Yu KN, Chang SH, Park SJ, Lim J, Lee J, et al. (2015) Titanium Dioxide Nanoparticles Induce Endoplasmic Reticulum Stress-Mediated Autophagic Cell Death via Mitochondria-Associated Endoplasmic Reticulum Membrane Disruption in Normal Lung Cells. PLoS One 10: 0131208.

63. Shi H, Magaye R, Castranova V, Zhao J (2013) Titanium dioxide nanoparticles: a review of current toxicological data. Part Fibre Toxicol 10: 15.

64. Monteiro-Riviere NA, Wiench K, Landsiedel R, Schulte S, Inman AO, et al. (2011) Safety evaluation of sunscreen formulations containing titanium dioxide and zinc oxide nanoparticles in UVB sunburned skin: an in vitro and in vivo study. Toxicol Sci 123: 264-280.

65. Zhao Y, Howe JL, Yu Z, Leong DT, Chu JJ, et al. (2013) Exposure to titanium dioxide nanoparticles induces autophagy in primary human keratinocytes. Small 9: 387-392.

66. Song B, Zhang Y, Liu J, Feng X, Zhou T, et al. (2016) Unraveling the neurotoxicity of titanium dioxide nanoparticles: focusing on molecular mechanisms. Beilstein J Nanotechnol 7: 645-654.

67. Zhang X, Yin H, Li Z, Zhang T, Yang Z (2016) Nano-TiO2 induces autophagy to protect against cell death through antioxidative mechanism in podocytes. Cell Biol Toxicol 32: 513-527.

68. Yu KN, Sung JH, Lee S, Kim JE, Kim S, et al. (2015) Inhalation of titanium dioxide induces endoplasmic reticulum stress-mediated autophagy and inflammation in mice. Food Chem Toxicol 85: 106-113.

69. Halamoda Kenzaoui B, Chapuis Bernasconi C, Guney-Ayra S, Juillerat-Jeanneret $L$ (2012) Induction of oxidative stress, lysosome activation and autophagy by nanoparticles in human brain-derived endothelial cells. Biochem $\mathrm{J}$ 441: 813-821.

70. Lin J, Huang Z, Wu H, Zhou W, Jin P, et al. (2014) Inhibition of autophagy enhances the anticancer activity of silver nanoparticles. Autophagy 10: 2006 2020.

71. Johnson BM, Fraietta JA, Gracias DT, Hope JL, Stairiker CJ, et al. (2015) Acute exposure to $\mathrm{ZnO}$ nanoparticles induces autophagic immune cell death Nanotoxicology 9: 737-748.

72. Aydin Sevinc B, Hanley L (2010) Antibacterial activity of dental composites containing zinc oxide nanoparticles. J Biomed Mater Res B Appl Biomater 94: 22-31.

73. Yu KN, Yoon TJ, Minai-Tehrani A, Kim JE, Park SJ, et al. (2013) Zinc oxide nanoparticle induced autophagic cell death and mitochondrial damage via reactive oxygen species generation. Toxicol In Vitro 27: 1187-1195.

74. Pati R, Das I, Mehta RK, Sahu R, Sonawane A (2016) Zinc-Oxide Nanoparticles Exhibit Genotoxic, Clastogenic, Cytotoxic and Actin Depolymerization Effects by Inducing Oxidative Stress Responses in Macrophages and Adult Mice. Toxicol Sci 150: 454-472.

75. Roy R, Singh SK, Chauhan LK, Das M, Tripathi A, et al. (2014) Zinc oxide nanoparticles induce apoptosis by enhancement of autophagy via PI3K/Akt/ mTOR inhibition. Toxicol Lett 227: 29-40. 